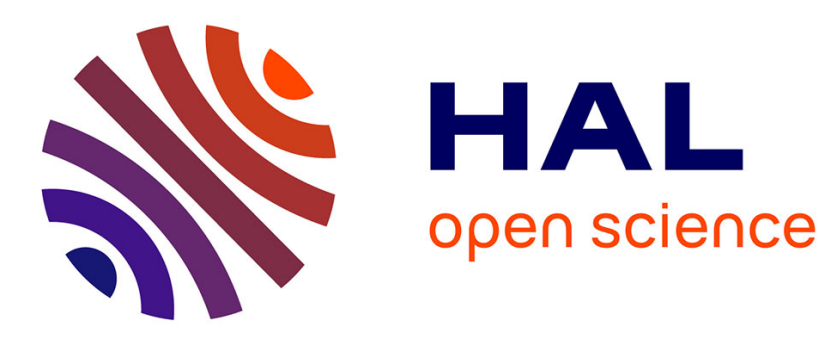

\title{
An Edge Computing Approach to Explore Indoor Environmental Sensor Data for Occupancy Measurement in Office Spaces
}

Sofiane Zemouri, Damien Magoni, Ayoub Zemouri, Yiannis Gkoufas, Kostas Katrinis, John Murphy

\section{To cite this version:}

Sofiane Zemouri, Damien Magoni, Ayoub Zemouri, Yiannis Gkoufas, Kostas Katrinis, et al.. An Edge Computing Approach to Explore Indoor Environmental Sensor Data for Occupancy Measurement in Office Spaces. IEEE International Smart Cities Conference, Sep 2018, Kansas City, United States. pp.1-8, 10.1109/ISC2.2018.8656753 . hal-02493482

\section{HAL Id: hal-02493482 \\ https://hal.science/hal-02493482}

Submitted on 27 Feb 2020

HAL is a multi-disciplinary open access archive for the deposit and dissemination of scientific research documents, whether they are published or not. The documents may come from teaching and research institutions in France or abroad, or from public or private research centers.
L'archive ouverte pluridisciplinaire HAL, est destinée au dépôt et à la diffusion de documents scientifiques de niveau recherche, publiés ou non, émanant des établissements d'enseignement et de recherche français ou étrangers, des laboratoires publics ou privés. 


\section{An Edge Computing Approach to Explore Indoor Environmental Sensor Data for Occupancy Measurement in Office Spaces}

\author{
Sofiane Zemouri \\ Univ. Coll. Dublin - Lero@PEL \\ Dublin, Ireland \\ Yiannis Gkoufas \\ IBM Research, Ireland \\ Dublin, Ireland
}

\author{
Damien Magoni \\ Univ. of Bordeaux - LaBRI \\ Bordeaux, France \\ Kostas Katrinis \\ IBM Research, Ireland \\ Dublin, Ireland
}

\author{
Ayoub Zemouri \\ Univ. of Boumerdes - Math. Dept. \\ Boumerdes, Algeria \\ John Murphy \\ Univ.Coll. Dublin-Lero@PEL \\ Dublin, Ireland
}

\begin{abstract}
Human occupancy measurement has become a topic of increasing interest in the past few years, due to the important role it plays in controlling a number of demand-driven applications like smart lighting and smart heating, as well as improving the energy efficiency of these applications in a broader sense. Office occupancy monitoring in commercial buildings can yield huge savings and improvements in terms of thermal, visual, and air quality. However, this is often impeded due to the lack of fine-grained occupancy information. This paper explores the use of low-priced environmental (temperature and humidity) sensor data for measuring occupancy in an office space. The idea behind this work is to leverage the variation divergence between humidity and temperature caused by human presence. We used a Raspberry Pi with a daughterboard called Sense Hat, which is equipped with the environmental sensors used in this study. The results are compared with occupancy data obtained from camera feeds in order to assess the effectiveness and the accuracy of the combined occupancy measurements, and show up to $87 \%$ accuracy.
\end{abstract}

Index Terms-Occupancy detection, occupancy measurement, RaspberryPi, Internet of Things, Edge Computing, IBM Cloud.

\section{INTRODUCTION}

Internet of Things (IoT) has become a major technological revolution, which is leading to an explosive growth in the number of internet-connected devices globally thanks to a strong reduction in sensors costs. Experts estimate that IoT will consist of approximately 50 billion devices by 2020 [1]. Consequently, IoT is gradually impacting every business area and industry field. These IoT devices offer an attractive, lowercost alternative to more expensive traditional monitoring systems like surveillance cameras or traditional weather stations. In addition, these devices can be more easily integrated with control systems like Building Management Systems (BMS) [2], thanks to their integrated communication modules.

The decrease in sensors prices was also accompanied with a continuous decrease in electronic components prices and

This research was partly funded by IBM Faculty Award grant, by Science Foundation Ireland grant 13/RC/2094 (Lero), by SFI grant 17/IFB/5401, and by ANR grant 10IDEX-03-02 to the University of Bordeaux. particularly a reduction in processing power costs. This led to more and more computational power being integrated into IoT devices, which paved the way to the advent of a new concept commonly called Edge Computing (EC) [3]. EC consists of pushing the intelligence of a system towards the edge of the network composing it. In other words, offloading some of the tasks that are usually handled on the cloud, to the some "cloudlets" [4] closer to the source of the data or to the IoT devices themselves.

The most notable advantage of such an approach is the considerable reduction of the response time enabling new, latency sensitive applications that were not possible using a traditional cloud architecture. Moreover, how effectively these IoT devices are able to communicate (particularly over potentially heavily congested networks) can have a major impact on the quality of the services provided, as well as in their degree of success (or failure). EC allows for a considerable reduction in the network load, since a good part of the data processing is done on the IoT device and thus, less data is transferred through the network to the cloud.

With the availability of IoT sensor data in commercial buildings, occupancy information can be monitored in real time. This information can be used in a variety of applications controlled by the BMS. For example, energy consumption can be regulated by controlling the Heating, Ventilating and Air Conditioning (HVAC) based on the occupancy information gathered from the sensors. In case of issues with HAVC, temporary adjustments can be made in order to ensure occupants comfort and safety [5], [6]. In addition to energy management, the fire system can benefit from such information. Survivors can be located and rescued in case of emergency situations [7]. Finally, occupancy information can be used to enhance the building security and surveillance [8].

In the recent years, many occupancy measurement approaches have been investigated in the literature to explore different flavours of IoT devices, sensor types combination, data processing methods, as well as functions and usecases. 
However, these approaches have some pros and cons in terms of cost, accuracy and privacy. This latter is a big concern especially in an office spaces where the privacy of occupants is of high importance. This restricts the use of a number of intrusive devices that can uncover the identity of the occupant or track their movements in the context of a commercial building or occupancy in office spaces. On the other hand, it is common sense that cost and accuracy are positively correlated as usually, the higher the accuracy of the sensor and its readings, the higher its cost is. Nevertheless, we argue that the accuracy of occupancy measurement could be improved relying on a combination of a number of different sensor readings.

This work investigates the use of low-cost, non-intrusive environmental sensors and the fusion of their readings in order to estimate office spaces occupancy more accurately in commercial buildings. We are particularly interested in detecting human presence in an office space as the number, location, and activity of the occupants are outside the scope of this work.

The main premise of this paper is that human presence in a closed space like an office, affects the temperature and the humidity in different ways, which creates some interesting divergences in the humidity and temperature behaviour between occupied and unoccupied periods. This difference in pattern is exploited to detect human presence.

First, the environmental sensor readings are gathered using the Sense Hat ${ }^{1}$. Next, these readings are combined and processed locally on the Raspberry $\mathrm{Pi}^{2}$ to detect changes in humidity and temperature patterns. Finally, the occupancy information in the office space is estimated based on the change in humidity and temperature behaviour. In order to assess the accuracy of our occupancy estimation method, we compared the occupancy results we obtained with manually reviewed video recordings of the office using a Pi Camera 3 . The proposed approach leverages the computational power of edge devices to alleviate the network load compared with traditional approaches where data is transferred to the cloud creating a bottleneck in the network.

The remainder of this paper is organized as follows: section II is a review of the current state of the art and a discussion of different approaches, their pros and cons. Section III introduces the method followed in order to infer occupancy in the office space. Section IV discusses the results and the limitations of this approach. Finally, section $\mathrm{V}$ discusses future directions and concludes this paper.

\section{RELATED WORK}

There are a number of occupancy measurement solutions in the literature. Throughout the past decade, researchers have explored various detection mechanisms using different types of sensors. Perhaps, the most obvious solution when trying to detect human presence is to use a system humans can

\footnotetext{
${ }^{1}$ https://www.raspberrypi.org/products/sense-hat/

${ }^{2}$ https://www.raspberrypi.org/

${ }^{3} \mathrm{https}: / /$ www.raspberrypi.org/products/camera-module-v2/
}

interact with to mark their presence, like the work in [9]. The work in [10] used carefully placed Passive Infra-Red (PIR) sensors to detect if someone cuts the infrared beam. A criticism of such solutions would be that they require a direct line of sight between the sensor and the occupant [11]. It also requires continuous movements from the occupants [12]. Other studies have demonstrated that such systems can be triggered by heat currents in the room, causing frequent false positives occurrences [13]. Other works relied on sound sensors [14] or ultrasonic sensors [15] to detect the noises caused by humans in a room or an office space. These solutions also fail to detect human presence if the person is not making any noises (for example a person sitting and silently reading an article). $\mathrm{CO}_{2}$ sensors were also explored in [16] to measure the change in the amount of $\mathrm{CO}_{2}$ exhaled by humans in a room. However, it is shown in works like [17] that the accuracy of such measurement with regards to human presence is much lower compared to other types of sensors. Another innovative solution aims to detect the Electromagnetic Signals (EM) produced by devices carried by the user like the work in [18] who monitors the Wi-Fi activity of a user. These solutions can be convenient, as they do not require any additional infrastructure, but they also come with a number of limitations. First, occupants have to carry a communication device (like a mobile phone) at all times. In addition, it is well known that mobile phones are battery powered and thus, can go offline at any time causing the system to fail at detecting a presence in the room [18]. Finally, Cameras are also a good way of obtaining fine-grained information about occupancy in a room or an office space like the works in [19] and [20]. Yet, there is a growing concern about occupants' privacy in office spaces [13]. Also, video feeds from cameras transferred through the network create a bottleneck in the network, hence the importance of EC.

These above occupancy measurement methods and others, can be classified according to a number of parameters. A good classification divides the literature works into three main categories [21]:

- Detection method: Occupancy measurement solutions can be classified according to the method used to detect the occupants in a building or in an office space. This can be an active or a passive detection. Active detection requires the occupant to explicitly perform an action to interact with the monitoring system and mark its presence. This usually involves a device or an object that needs to be carried by the user such as an access card or an RFID tag like the work in [9]. Although this method allows for a great level of accuracy in perfect conditions, it is dependent on the occupants' compliance. On the other hand, passive detection does not require any actions from the user. Most of the solutions mentioned above (EM, CO2, PIR, ultrasonic and cameras) fall into this category, as they are very convenient. However, it is argued in many works [17] that the accuracy of such solutions relying on a single source of information can 


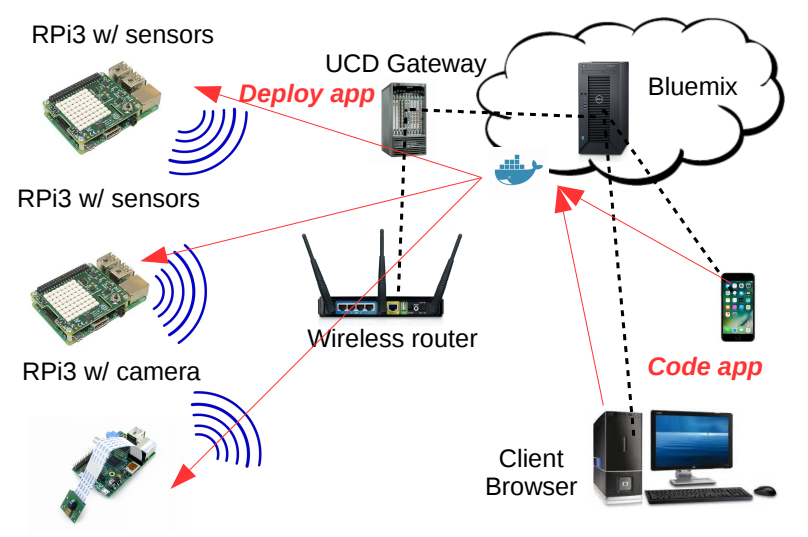

Fig. 1. IoT testbed used in this study

be improved by using a fusion of a number of different sensor data.

- Detection Function: Occupancy measurement solutions can also be classified according to the function or the purpose of the detection. The function of the detection can range between presence, count, location, movement tracking and activity monitoring. Presence detection provides binary information (true or false) about whether the room is occupied or not [18]. Count measurement on the other hand gives information about the number of persons present in a room, an office space or a building [13]. Location detection allows the HVAC system to adjust in specific locations according to where persons are in a building [16]. Movement tracking takes this a step further and provides the history of a particular occupant's movement [22]. Finally, activity monitoring is concerned with what the occupant is doing like the work in [23], which relies on $\mathrm{CO} 2$ exhaled by human presence to determine the level of effort of different individuals. However, there are a lot of privacy concerns about solutions like the above that track occupants' location, movements and activity [13].

- Detection Infrastructure: The last classification divides the literature works based on whether they require the installation of explicit infrastructure to operate, or they just rely on existing infrastructure that performs the occupancy measurement as a secondary function [24], [25]. CO2, PIR, and ultrasonic sensors are explicit solutions, as they require new sensors to be installed in the office space. On the other hand, solutions that rely on EM measurements or video feeds can be regarded as implicit solutions as they infer occupancy information from sources that are not solely installed for the purpose of this detection.

A common trait of all the works discussed in this section is that most of the time they rely on a single source of information. The novelty of this work is the fact that it looks into different measurements sources from low-cost environ- mental sensors and combines them in order to improve the accuracy of the detection. The use of such sensors eliminates all privacy concerns, as these readings do not allow unveiling the identity of the occupants. Another important advantage of our solution is the use of EC to process and aggregate the sensor readings locally, which considerably reduces the amount of data injected in the network.

\section{Proposed Solution}

In this section, we will first go through the system design, explain the testbed and the setup used in this study, then we will cover the approach followed to estimate the occupancy in an office and the steps involved.

\section{A. System Design}

For the purpose of this work, we have developed an IoT test bed at University College Dublin (UCD) to detect human presence in a few approved offices. We relied on the cloud IoT platform IBM Bluemix ${ }^{4}$ to provision the IoT devices with the packaged code. This platform uses Docker containers ${ }^{5}$ to package the code which allows for a seamless provision and update of the Edge Devices. It also offers a dashboard that allows a holistic view and an easier interaction with the devices and the data they report. We refer the reader to [26] for more information about IBM Bluemix.

The IoT test bed used in this study is illustrated in Figure 1. The test bed comprises of a number of Raspberry Pi's System on Chip (SoC) as Edge Devices. These devices are connected to a wireless router, which in turn is linked to a gateway allowing them to have Internet access and connect to IBM Bluemix.

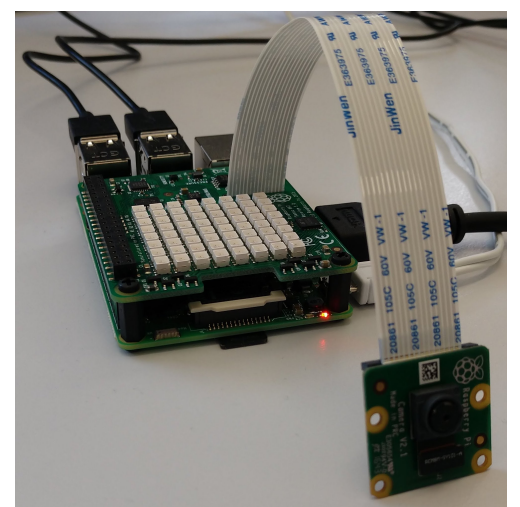

Fig. 2. Edge device used in this study (Raspberry Pi3 equipped with a Sense Hat daughterboard and a Pi Camera)

The Edge devices are hooked with a daughterboard called Sense Hat that contains six sensors in total. These sensors can be classified in two types: (i) Environmental sensors, which include Temperature, Humidity and Barometric Pressure sensors; and (ii) Inertial sensors comprising in a Compass, a Gyroscope and an accelerometer. The inertial sensors were

\footnotetext{
${ }^{4}$ https://www.ibm.com/cloud/

${ }^{5}$ https://www.docker.com/
} 


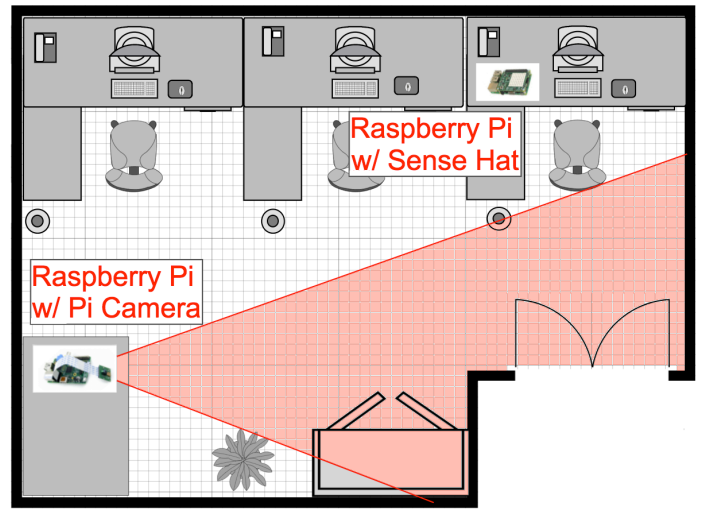

Fig. 3. Layout of the test office used in this study and location of Edge Devices

not used for the purpose of this work as the Edge Device was stationary in our case. We also equipped one Raspberry Pi with a Pi Camera in order to assess the accuracy of the detection using the above-mentioned environmental sensors. This was done by manually checking the video recordings and obtaining the baseline real occupancy information, then comparing them against the sensor readings. Figure 2 shows an Edge Device used in this study.

The edge devices were placed as shown in Figure 3 in a test office. The office comprises three desks. The Raspberry $\mathrm{Pi}$ equipped with a camera was placed in a way to capture employees coming in and out of the office. Another Raspberry Pi equipped with a Sense Hat was placed on one of the desks to capture environmental (humidity and temperature) data in the office. In the next subsection, we describe the approach and the different methods to probe occupancy information in the office space.

\section{B. Approach}

As mentioned in the previous sections, the Sense Hat contains three environmental sensors (Temperature, Humidity and Barometric Pressure), as well as three other inertial sensors (Gyroscope, Compass and Accelerometer). After inspecting a sample dataset of all these sensors' readings, we came to the conclusion that three out of the six sensors did not yield useful enough information for this specific application, as there was barely any variation in the measured values. These sensors are the gyroscope, the accelerometer and the magnetometer. This was expected since these three inertial sensors are designed to capture movements in the edge device. However, this later was stationary in our case. In the environmental sensors category, the Barometric pressure sensor was also dropped as it did yield some variations, but these were long-term trends rather than the instantaneous spikes we are interested in. Based on these elements, we only relied on the humidity and the temperature sensors in our study.

The main finding of this work is the fact that human presence in a closed room affects the behaviour of the humidity and the temperature in different ways. In fact, in a closed, undisturbed environment, temperature and humidity vary as a pair. That is, usually an increase in the temperature is always accompanied with a decrease in humidity, and vice versa [27]. As a person walks into the room, this equilibrium is disturbed to a certain extent and we observe a different pattern where the changes in temperature and humidity have different amplitudes.

In the following, we will go through the approach and the steps followed to result in the occupancy detection solution proposed in this work. This can be described in the following three steps: (i) data smoothing and de-trending; (ii) pattern observation and data comparison; and finally (iii) occupancy estimation. We will discuss each of these steps and justify their significance and the logic behind them.

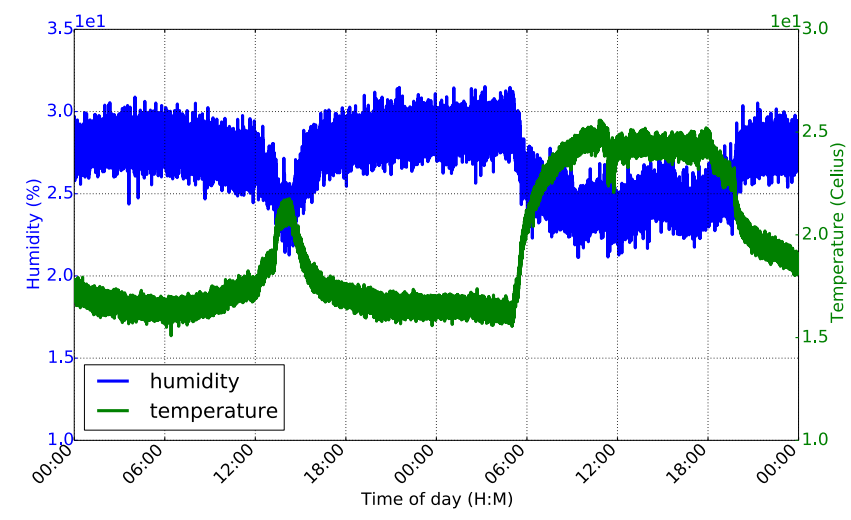

(a) Raw temperature and humidity

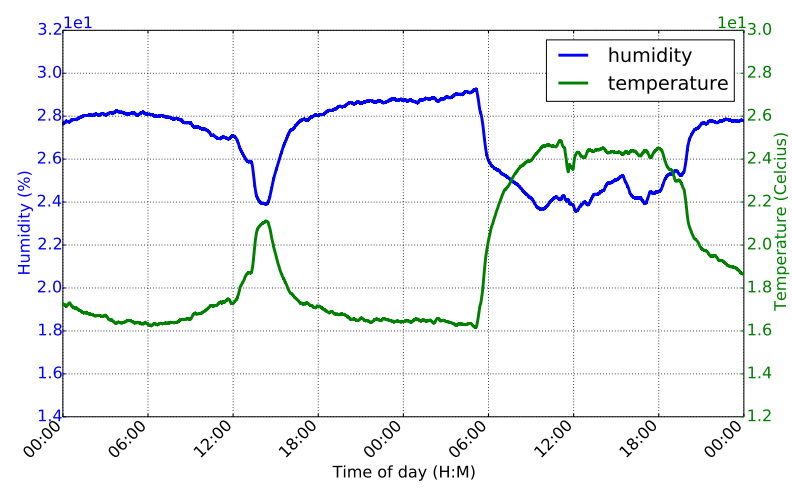

(b) EWMA Smoothed temperature and humidity

Fig. 4. difference between raw and smoothed humidity and temperature data. EWMA with a small enough decay factor allows removing the noise while maintaining the shape of the data.

(i) Data smoothing and de-trending: In this first step, we applied an Exponentially Weighted Moving Average (EWMA) to smooth the data and get rid of the unnecessary noise. EWMA is calculated as follows:

$$
a_{t}=(1-\lambda) \sum_{i=0}^{t-1} \lambda^{i} a_{t-1-i}
$$

Where $a_{t}$ is the exponentially weighted sample at time $t$ and $\lambda$ is the decay parameter. The choice of EWMA 
against a Simple Moving Average (SMA) is motivated by the fact that we wanted to smooth the data but preserve variations and the spikes observed in the data by giving more weight to more recent data. In other words, an EWMA allows smoothing the data while maintaining the integrity or the shape of the data whereas a SMA not only smooths the data, but it also considerably reduces the magnitude of the spikes observed in the data (or smooth the edges). Figure 4 shows a sample temperature and humidity plot before and after applying the EWMA.

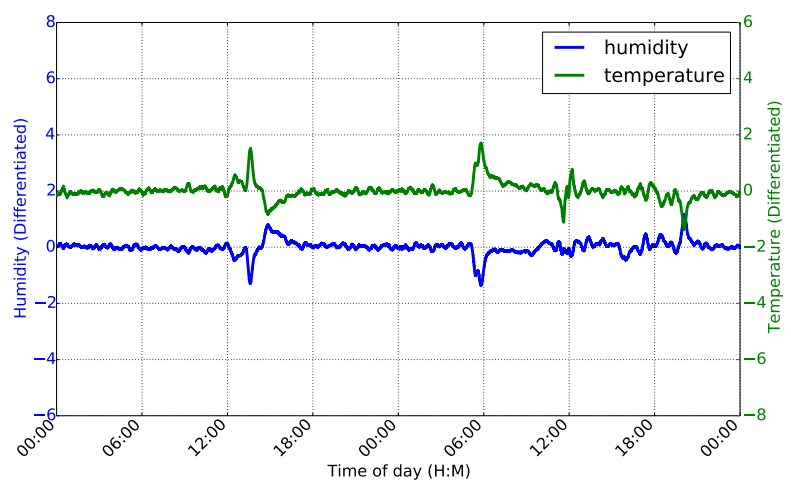

(a) Differentiated temperature and humidity data

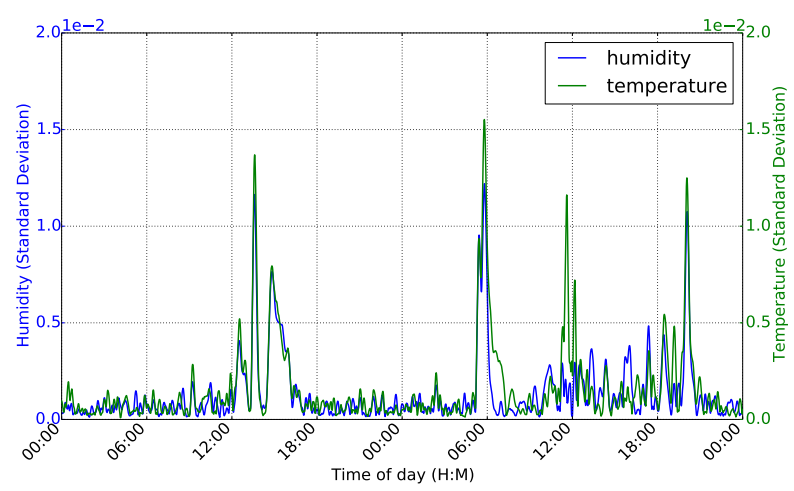

(b) Standard Deviation of differentiated humidity and temperature data

Fig. 5. De-trending using numerical differentiation (a) then quantification of the variation (b) in order to infer patterns of occupied and unoccupied periods.

After the EWMA is applied, we performed a rolling numerical differentiation of the data using the following formula:

$$
\Delta\left(a_{t}\right)=a_{t}-a_{t-w}
$$

Where $a_{t}$ is the term at time $t$ and $w$ is the time window of the differentiation. The reason behind this step is the elimination of trends and seasonality. In fact, temperature (and consequently humidity) changes throughout the day in account of external factors such as outdoor temperature, as well as the heating cycle in the room. However, we are only interested in variations caused by human presence and thus, numerical differentiation is the perfect candidate to filtering out the long-term variations while maintaining the integrity of short-term temperature and humidity changes. Figure 5(a) shows the output of differentiated temperature and humidity data.

Finally, we calculated the standard deviation of this differentiated data as follows:

$$
\sigma\left(a_{t}\right)=\sqrt{\frac{1}{t-1} \sum_{i=1}^{t}\left(a_{i}-\bar{a}(w)\right)^{2}}
$$

Where $\bar{a}(w)$ is a rolling mean with a window $w$. By definition, the standard deviation is the amount of change from the mean value. In our study, we wanted to quantify the amount of variation in the differentiated data. In other words, this allows us to measure the difference in humidity and temperature variations and draw a clear picture of whether there is a correlation between these two values. Figure 5(b) shows the resulted standard deviation.

(ii) Pattern observation and data comparison: For the sake of simplicity, we will refer to the Standard Deviation of the differentiated data as $\sigma$. In this step, we divided the data into two groups: occupied and unoccupied periods. Real occupancy information was obtained by means of reviewing recorded videos and photos of the test office, recording the time and mapping this information to our humidity and temperature $\sigma$ values. We plotted samples of occupied periods as well as unoccupied periods and the results are shown in Figure 6.
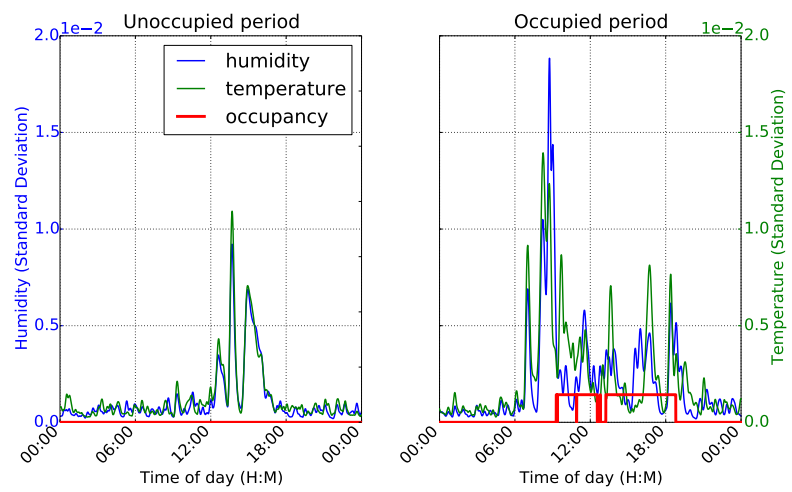

Fig. 6. Difference in Temperature and Humidity Standard Deviation between occupied (right) and unoccupied (left) periods.

The graphs show an interesting pattern, that is, humidity and temperature $\sigma$ values follow the same behaviour during unoccupied periods, but diverge significantly during occupied periods. Based on this observation, we wanted to confirm this observation and extract useful occupancy information from it.

In order to do this, we calculated the absolute difference between the humidity and temperature $\sigma$ values to get a better feel of the convergence and divergence observed using the following formula: 

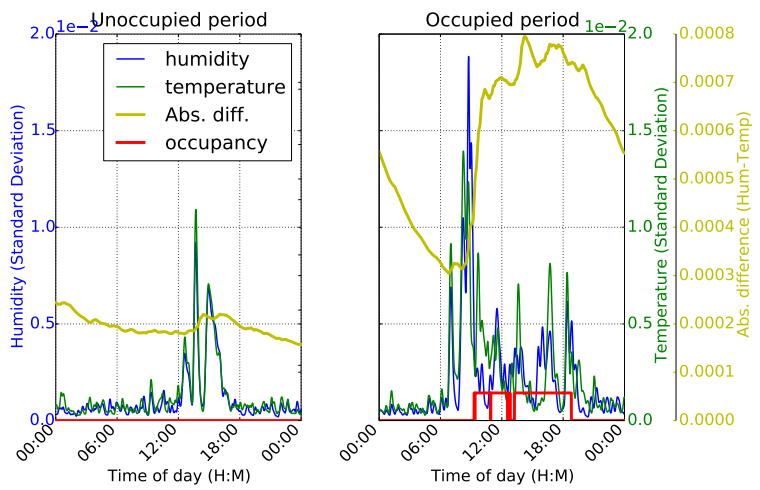

Fig. 7. Absolute difference of Humidity and Temperature Sigma versus occupancy.

$$
\rho_{t}=\left|\sigma\left(T_{t}\right)-\sigma\left(H_{t}\right)\right|
$$

Where $\sigma\left(T_{t}\right)$ is the Standard Deviation of the differentiated temperature at time $t$ and $\sigma\left(H_{t}\right)$ is the Standard Deviation of the differentiated humidity at time $t$. After smoothing this $\rho_{t}$ by applying an EWMA filter from Equation 1, we obtained the graph shown in Figure 7.

From this figure, we start to see a clear pattern where $\rho$ value increases significantly during occupancy periods and drops rapidly when the person leaves the office. On the other hand, $\rho$ remains fairly flat when the room is empty for a long time and is little affected by the sudden spikes in humidity or temperature caused by external factors like the heating system or the outside temperature.

(iii) Occupancy estimation: In this final step, we apply an estimation algorithm based on $\rho_{t}$ calculated in the previous step. The input is simply the differentiation of this value. This is done to quantify how fast this measure is varying and is calculated as follows:

$$
\Delta\left(\rho_{t}\right)=\rho_{t}-\rho_{t-w}
$$

Where $\rho_{t}$ is the value at time $t$ and $w$ is the time window of the differentiation. The output on the other hand is the estimated occupancy information. The algorithm is intuitive and pretty straightforward and is shown in Algorithm 1.

Three parameters are used for the purpose of the estimation and that is the Ascending Velocity Threshold $A s c_{t h}$, the Descending Velocity Threshold $D e s c_{t h}$ and the Decline Threshold $D e c_{t h}$. The former two parameters are self-explanatory as they are thresholds for how fast the $\Delta(\rho)$ (Equation 5) is increasing or decreasing. If $\Delta(\rho)$ calculated above is smaller than $D e s c_{t h}$, this means that the value is dropping rapidly and the state of the occupancy in the office should be changed to unoccupied. On the other hand, if $\Delta(\rho)$ is greater than $A s c_{t h}$, this is interpreted as a rapid increase in this value and thus, the occupancy state should be changed to occupied. The last parameter $\left(D e c_{t h}\right)$ on the other hand, which is the decline threshold, is a threshold to determine how long should $\Delta(\rho)$ value continuously drop in a moderate amount in order to consider that the office has been cleared and the occupancy status should be changed from occupied to unoccupied.

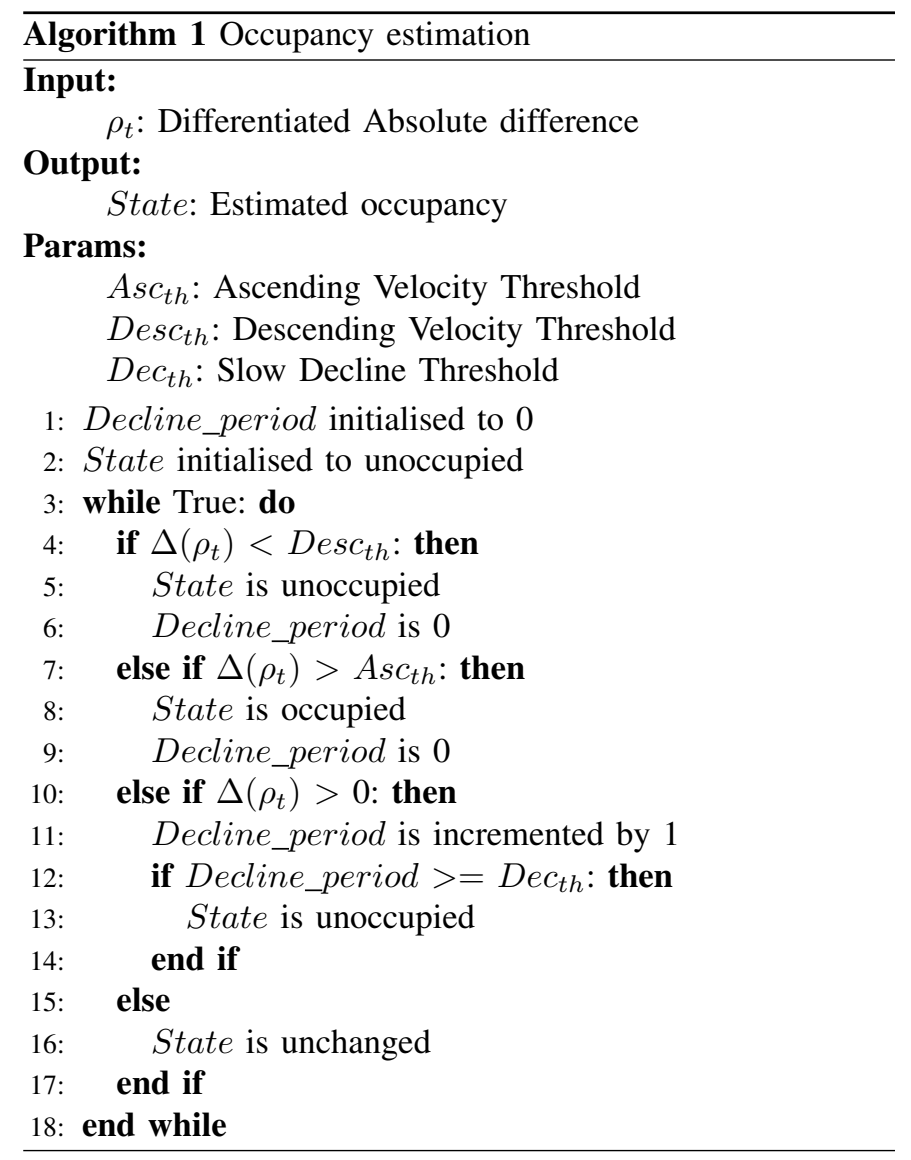

To sum the steps above up, first the raw humidity and temperature measurements are smoothed and de-trended using a series of EWMA, Differentiation, and Standard Deviation. We referred to this as $\sigma$. Next, the absolute difference between the humidity value $\sigma(H)$ and the temperature value $\sigma(T)$ is calculated and smoothed using an EWMA filter. We referred to this as $\rho$. A numerical differentiation of this parameter was performed next and referred to as $\Delta(\rho)$. Finally, the intuitive algorithm above is applied to estimate the occupancy in the room.

This method has the advantage of being extremely light and only relies on a relatively short window of past data. This allows such estimation to run on low cost edge devices in real-time without encountering any performance issued. In the following section, we will discuss the results we obtained using this method to estimate occupancy in an office space.

\section{RESULTS}

In order to prove the effectiveness of our solution, we used the setup described in Subsection III-A and gathered ten 


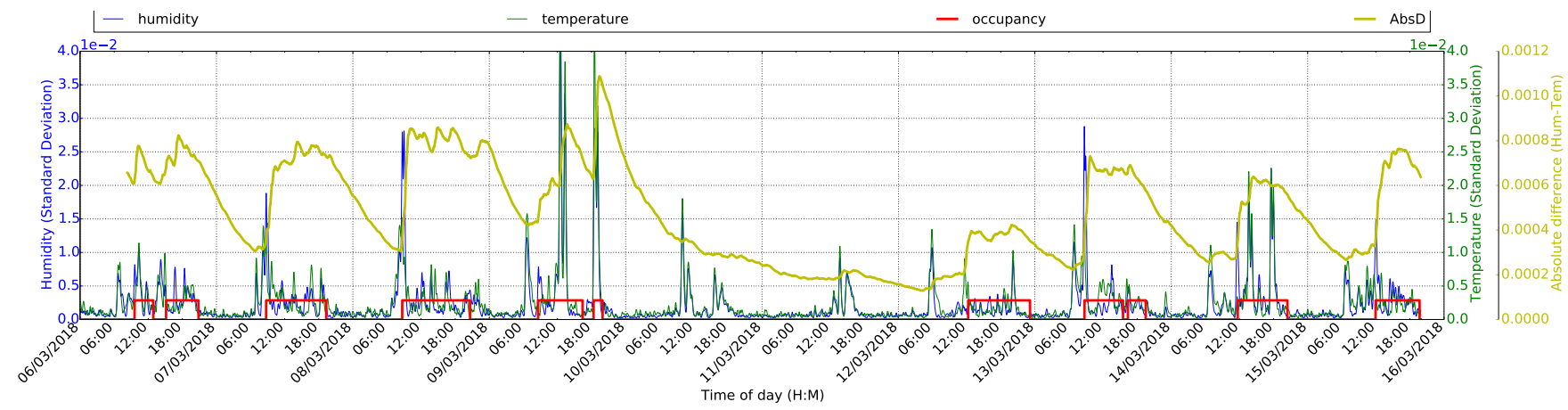

Fig. 8. Estimated occupancy inferred using the method described in Subsection III-B ( $D e c_{t h}$ of 8100 seconds).

days worth of humidity and temperature data. This dataset comprises of $860 \mathrm{~K}$ humidity and temperature measurements taken in 1 second intervals. Table I shows the empirically selected parameters used in our estimation. The decay factor $\lambda$ of the EWMA is the parameter used in the first step in Subsection III-B above. The window size $w$ is the rolling window used to calculate the numerical differentiation as well as the rolling mean of the Standard Deviation in the same step. Finally, $A s c_{t h}, D e s c_{t h}$, and $D e c_{t h}$ are the parameters described in Algorithm 1.

TABLE I

ESTIMATION PARAMETERS

\begin{tabular}{ccc}
\hline$\lambda$ & EWMA decay factor & 0.95 \\
\hline$A s c_{t h}$ & Ascending Velocity Threshold & $98 e^{-8}$ \\
$D e s c_{t h}$ & Descending Velocity Threshold & $62 e^{-8}$ \\
$D e c_{t h}$ & Slow Decline Threshold & $8100 \mathrm{sec}$ \\
\hline$w$ & Differentiation and & $900 \mathrm{sec}$ \\
& Standard Deviation window & \\
\hline
\end{tabular}

Our occupancy estimation approach was used and compared against real occupancy information gathered from video recordings. Figure 8 shows the results obtained after performing our occupancy estimation method over a period of ten days. This figure comprises of $\sigma(T)$ and $\sigma(H)$ (Standard Deviation of the differentiated temperature and humidity data respectively, Equation 3), as well as $\rho$ (absolute difference of $\sigma(T)$ and $\sigma(H)$ ) and the estimated occupancy information. In order to evaluate these results, we compared the estimated occupancy information with the real occupancy values and the resulting graph is shown in Figure 9. We can see from this figure that there our estimation method performs well relatively with the size of the data. This shows the potential of such an approach in detecting human presence in a room.

In order to give more insight into the results, we selected five evaluation metrics in order to assess the correctness of our estimation results. These metrics are (i) True Positive Rate; (ii) True Negative Rate; (iii) False Positive Rate; (iv) False Negative Rate; and finally (v) the Accuracy of the estimation. True Positive Rate and True Negative Rate are defined as $T P R=T P /(T P+F N)$ and $T N R=T N /(T N+F P)$ respectively; where TP, TR, FP and FR are the total number of True Positive, True Negative, False Positive and False Negative samples respectively. False Positive and False Negative Rates are calculated respectively as follows: $F P R=1-T N R$ and $F N R=1-T P R$. Finally, the accuracy of the estimation is obtained by $A C C=(T P+T N) /(T P+F P+T N+F N)$. We also varied the $D e c_{t h}$ parameter and obtained a set of results that are broken down in Table II.

TABLE II

ESTIMATION RESULTS

\begin{tabular}{l|llll|l}
\hline$D e c_{t h}$ & TPR & TNR & FPR & FNR & Accuracy \\
\hline $3600 \mathrm{~s}$ & $59.07 \%$ & $91.97 \%$ & $8.03 \%$ & $40.93 \%$ & $83.33 \%$ \\
$6900 \mathrm{~s}$ & $80.50 \%$ & $88.14 \%$ & $11.86 \%$ & $19.50 \%$ & $86.14 \%$ \\
$8100 \mathrm{~s}$ & $86.99 \%$ & $87.04 \%$ & $12.96 \%$ & $13.01 \%$ & $87.03 \%$ \\
$9900 \mathrm{~s}$ & $93.40 \%$ & $83.09 \%$ & $16.91 \%$ & $6.60 \%$ & $85.80 \%$ \\
$13300 \mathrm{~s}$ & $95.04 \%$ & $80.06 \%$ & $19.94 \%$ & $4.96 \%$ & $83.99 \%$ \\
\hline
\end{tabular}

By varying the $D e c_{t h}$ parameter (which describes how long should $\Delta(\rho)$ monotonically descend before the state of the room is changed from occupied to unoccupied), we observe that a higher $D e c_{t h}$ increases the True Positive Rate while a lower $D e c_{t h}$ increases the True Negative Rate. The accuracy of the estimation is a tradeoff between the True Positive Rate and the True Negative Rate. We should mention that this particular dataset has more instances of unoccupied states and therefore, a small change in the True Negative Rate affects the overall Accuracy greatly while an important change in the True Positive Rate does not affect the overall accuracy in such an important way. We also noted that varying the other parameters (for example the window size $w$ ) does not have as a great effect on the accuracy as the $D e c_{t h}$.

\section{CONClusion}

This paper explores an Edge Computing approach to use low-cost, non-intrusive environmental sensors for measuring occupancy in an office space. Humidity and temperature data was combined and used in order to estimate human presence in a test office. The main idea was to leverage the divergence in humidity and temperature variations introduced by human presence in a closed environment such as an office. The results show that such fusion of data (humidity and temperature) can help estimate room occupancy with up to $87 \%$ accuracy. This work is a demonstration that combination of different 


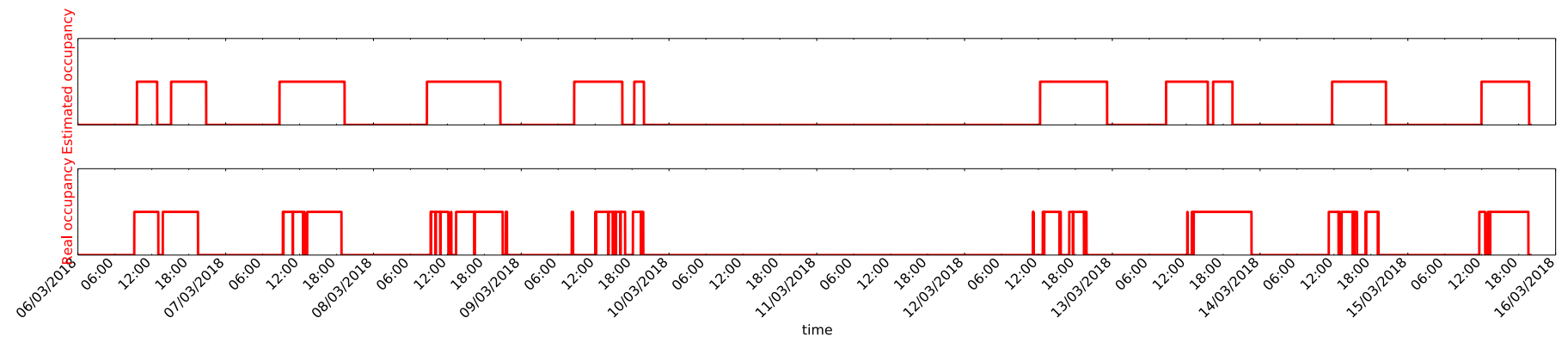

Fig. 9. Comparison between the estimated occupancy and the real occupancy with a $D e c_{t h}$ value of 8100 seconds.

types of sensor readings can yield more accurate occupancy information, and paves the way for more research in the data fusion research applied in the context of Smart Cities. Future work will explore the use of inertial sensor readings and fusion with environmental sensors to improve the accuracy of the estimation.

\section{REFERENCES}

[1] Evans, D., 2011. "The internet of things: How the next evolution of the internet is changing everything." CISCO white paper, 1(2011), pp.1-11.

[2] Levermore, G., 2013. "Building energy management systems: An application to heating, natural ventilation, lighting and occupant satisfaction." Routledge.

[3] Shi, W. and Dustdar, S., 2016. "The promise of edge computing." textitComputer, 49(5), pp.78-81.

[4] Lewis, G., Echeverria, S., Simanta, S., Bradshaw, B. and Root, J., 2014, October. "Tactical cloudlets: Moving cloud computing to the edge." In Military Communications Conference (MILCOM), 2014 IEEE (pp. 14401446). IEEE.

[5] De Paola, A., Ortolani, M., Lo Re, G., Anastasi, G. and Das, S.K., 2014 "Intelligent management systems for energy efficiency in buildings: A survey." ACM Computing Surveys (CSUR), 47(1), p.13.

[6] Nguyen, T.A. and Aiello, M., 2013. "Energy intelligent buildings based on user activity: A survey." Energy and buildings, 56, pp.244-257.

[7] Nyarko, K. and Wright-Brown, C., 2013, November. "Cloud based passive building occupancy characterization for attack and disaster response." In Technologies for Homeland Security (HST), 2013 IEEE International Conference on (pp. 748-753). IEEE.

[8] Akkaya, K., Guvenc, I., Aygun, R., Pala, N. and Kadri, A., 2015, March. "IoT-based occupancy monitoring techniques for energy-efficient smart buildings." In Wireless Communications and Networking Conference Workshops (WCNCW), 2015 IEEE (pp. 58-63). IEEE.

[9] Martani, C., Lee, D., Robinson, P., Britter, R. and Ratti, C., 2012. "ENERNET: Studying the dynamic relationship between building occupancy and energy consumption." Energy and Buildings, 47, pp.584-591.

[10] ul Haq, M.A., Hassan, M.Y., Abdullah, H., Rahman, H.A., Abdullah, M.P., Hussin, F. and Said, D.M., 2014. "A review on lighting control technologies in commercial buildings, their performance and affecting factors." Renewable and Sustainable Energy Reviews, 33, pp.268-279.

[11] Teixeira, T., Dublon, G. and Savvides, A., 2010. "A survey of humansensing: Methods for detecting presence, count, location, track, and identity." ACM Computing Surveys, 5(1), pp.59-69.

[12] Meyn, S., Surana, A., Lin, Y., Oggianu, S.M., Narayanan, S. and Frewen, T.A., 2009, December. "A sensor-utility-network method for estimation of occupancy in buildings." In Decision and Control, 2009 held jointly with the 2009 28th Chinese Control Conference. CDC/CCC 2009. Proceedings of the 48th IEEE Conference on (pp. 1494-1500). IEEE.

[13] Li, N., Calis, G. and Becerik-Gerber, B., 2012. "Measuring and monitoring occupancy with an RFID based system for demand-driven HVAC operations." Automation in construction, 24, pp.89-99.
[14] Uziel, S., Elste, T., Kattanek, W., Hollosi, D., Gerlach, S. and Goetze, S., 2013, October. "Networked embedded acoustic processing system for smart building applications." In Design and Architectures for Signal and Image Processing (DASIP), 2013 Conference on (pp. 349-350). IEEE.

[15] Tarzia, S.P., Dick, R.P., Dinda, P.A. and Memik, G., 2009, September. "Sonar-based measurement of user presence and attention." In Proceedings of the 11th international conference on Ubiquitous computing (pp. 89-92). ACM.

[16] Nassif, N., 2012. "A robust CO2-based demand-controlled ventilation control strategy for multi-zone HVAC systems." Energy and buildings, 45 , pp.72-81.

[17] Pedersen, T.H., Nielsen, K.U. and Petersen, S., 2017. "Method for room occupancy detection based on trajectory of indoor climate sensor data." Building and Environment, 115, pp.147-156.

[18] Khoury, H.M. and Kamat, V.R., 2009. "Evaluation of position tracking technologies for user localization in indoor construction environments." Automation in Construction, 18(4), pp.444-457.

[19] Erickson, V.L., Achleitner, S. and Cerpa, A.E., 2013, April. "POEM Power-efficient occupancy-based energy management system." In Proceedings of the 12th international conference on Information processing in sensor networks (pp. 203-216). ACM.

[20] Benezeth, Y., Laurent, H., Emile, B. and Rosenberger, C., 2011. "Towards a sensor for detecting human presence and characterizing activity." Energy and Buildings, 43(2-3), pp.305-314.

[21] Labeodan, T., Zeiler, W., Boxem, G. and Zhao, Y., 2015. "Occupancy measurement in commercial office buildings for demand-driven control applications - A survey and detection system evaluation.” Energy and Buildings, 93, pp.303-314.

[22] Klein, L., Kwak, J.Y., Kavulya, G., Jazizadeh, F., Becerik-Gerber, B., Varakantham, P. and Tambe, M., 2012. "Coordinating occupant behavior for building energy and comfort management using multi-agent systems." Automation in construction, 22, pp.525-536.

[23] Dougan, D.S. and Damiano, L., 2004. "CO2-based demand control ventilation: Do risks outweigh potential rewards?." ASHRAE Journal, $46(10)$, p. 47.

[24] Thanayankizil, L.V., Ghai, S.K., Chakraborty, D. and Seetharam, D.P., 2012, January. "Softgreen: Towards energy management of green office buildings with soft sensors." In Communication Systems and Networks (COMSNETS), 2012 Fourth International Conference on (pp. 1-6). IEEE.

[25] Christensen, K., Melfi, R., Nordman, B., Rosenblum, B. and Viera, R., 2014. "Using existing network infrastructure to estimate building occupancy and control plugged-in devices in user workspaces." International Journal of Communication Networks and Distributed Systems, 12(1), pp.4-29.

[26] Gheith, A., Rajamony, R., Bohrer, P., Agarwal, K., Kistler, M., Eagle, B.W., Hambridge, C.A., Carter, J.B. and Kaplinger, T., 2016. "Ibm bluemix mobile cloud services." IBM Journal of Research and Development, 60(2-3), pp.7-1.

[27] Williams, R., Rankin, N., Smith, T., Galler, D. and Seakins, P., 1996. "Relationship between the humidity and temperature of inspired gas and the function of the airway mucosa." Critical care medicine, 24(11), pp.1920-1929. 